\title{
STUDY OF ANTIBIOTIC RESISTANCE ON ESCHERICHIA COLI IN COMMERCIAL POULTRY OF NEPAL
}

\author{
T. Khanal ${ }^{*}$, S. B. Raut ${ }^{2}$ and U. Paneru ${ }^{3}$ \\ ${ }^{1 *}$ Agriculture and Forestry University, Chitwan, Nepal \\ ${ }^{2}$ Nepal Agricultural Research Council, Lumle, Pokhara, Nepal \\ (*email: tankakhanala01@gmail.com)
}

\begin{abstract}
The continuous use of antibiotics in compound feed at sub-therapeutic level has been an integral part of commercial poultry production in Nepal, which is one of the factors that promotes bacterial resistance. Hence, with the objective to determine antibiotic resistance in commercial poultry of Nepal, this study was designed taking Escherichia coli as a flagship bacterium. The commercial layers and broilers birds brought to veterinary teaching hospital of Agriculture and Forestry University by commercial poultry producers for disease diagnosis and treatment were considered as clinical examination of birds were carried out followed by post mortem examination (PME). Those layer/broiler birds which were not taking antibiotic orally or parenterally for last 2 weeks and diagnosed with collibacillosis on PME were included in sampling frame. Air sacculitis, fibrinous pericarditis, fibrinous perihepatitis, and coligranuloma were major criteria for presumptive diagnosis of colibacillosis on PME. The first 40 for both broiler and layer birds totaling 80 that fulfilled the criteria were selected as samples, each representing a commercial farm. All necessary information on daily management practices and previous treatments were obtained from farmer's record book or sheets or face to face interview. Avian pathogenic E. coli was isolated from aseptically collected liver samples and confirmedby biochemical tests. Antibiogram of the isolates were investigated by means of Kirby-Bauer disc diffusion method. E. coli was isolated from all liver samples taken for the culture. It was found that E. coli were resistant most substantially towards Cephalexin (81.2\%) and Amoxycillin (81.2\%) followed by Tetracycline (78.8\%), Colistin sulphate $(n=50,62.5 \%)$. Chloramphenicol (61.2\%), Ciprofloxacin (55.0\%), Enrofloxacin (53.8\%), Levofloxacin (28.8\%), however, no resistance was found against amikacin. The proportion of E. coli isolates that were resistance against Colistin sulphate $(p<0.05)$, Chloramphenicol $(p<0.05)$, Tetracycline $(p<0.001)$, Ciprofloxacin $(p<0.01)$, Enrofloxacin $(p<0.05)$ and Gentamicin $(p<0.01)$ were significantly higher in layers compared to that of broilers. In conclusion, avian pathogenic E. coli were resistant towards several antibiotic molecules commonly used in commercial poultry of Nepal, and the resistance was higher in layers compared to broilers.
\end{abstract}

Keywords: antibiotic resistance, E. coli, poultry 


\section{INTRODUCTION}

The use of antibiotics in compound feeds has been an integral part of poultry production, not only to prevent infectious bacterial diseases but also to promote growth of host. Almost all poultry feed industry in Nepal commonly use different molecules of antibiotics as feed additives in compound feed, pelleted or mash, with the objective to enhance growth and feed efficiency. This continuous use of several types of antibiotic molecules at sub therapeutic level in feeds promotes the bacterial resistance in poultry (Aryal, 2001). The bacteria acquire resistance to particular class of antibiotic either due to de novo mutation, or due to transmission of acquired resistance from one bacterium to others, within species or between species via extra chromosomal DNA (Tenover, 2006). Resistant bacteria thwart antibiotics by interfering their mode of action such as synthesis of inactivating enzymes, alteration in configuration of cell wall or ribosome, and modification of membrane carrier systems, (Timoney et al., 1988; Prescott and Dowling, 2013), inhibition of nucleic acid synthesis, inhibition of metabolic pathway (Tenover, 2006). The widespread use of antibiotics as feed additives for growth promotion or disease prevention in food animals could have negative implications for human health and the environment (Hasan et al., 2011).

They also noted a common way of transmission of resistant clones and resistance plasmids of $E$. coli from poultry to human. The development of antibiotic resistance in human bacteria and its association with sub-therapeutic use of antibiotic in animal feed were discussed since early 70's (Dibner and Richards, 2005). The E. coli resistant isolates from poultry in a region plays important role for human E. coli infections in that region as Agabou et al. (2016) showed a clonal and epidemiological link between chicken and human ciprofloxacin-resistant $E$ coli isolates in Algeria. Furthermore, the intestine of poultry consists zoonotic potential E. coli, which can be transferred from birds to human (Ewers et al., 2009). The continuous use of antibiotics in compound feed creates a selective pressure to develop resistant $E$. coli in poultry gut that could make its way in human food chain (Diarra et al., 2007). Additionally, in birds, the resistance transferred from generation to generation as pyramid. In a recent study at Sweden, scientists reported occurrence of E. coli resistant to nalidixic acid in grandparent birds imported to Sweden for breeding purpose and resistance transferred to parents and their broilers. Similarly, quinolones resistant $E$ coli can be introduced from imported breeding birds and spread by vertical transmission (Borjesson et al., 2016).

In Nepal, monitoring of antibiotic resistance in veterinary medical field started very late (Khatiwada, 2011) and only a limited number of papers have been published. Antibiogram study of $E$. coli isolated in Nepal from apparently healthy and diarrheic ducks were studied by Singh et al., (2013). Shrestha et al., (2010) first reported a finding on antimicrobial resistance of Salmonella in poultry of Nepal. In human medical field in Nepal, a couple of studies have been conducted to understand antibiogram of $E$. coli isolated form patients of urinary tract infection (UTI). Sharma et al., (2013) and Baral et al., (2012) found E. coli as one of the major causes of UTI. Majority of E. coli showed a resistance towards ampicillin, cephalexin, Nalidixic acid and ciprofloxacin and are multidrug resistant. 
In most of the researches, antimicrobial resistance profile of $E$. coli is studied in apparently healthy poultry where $E$. coli are isolated from faecal samples. However, in this research, the antimicrobial resistance was studied in birds which suffered from colibacillsis and E. coli was isolated from liver but not from faeces. In this sense, this research is unique and novel. Here, the focus is on the antimicrobial resistance pattern of $E$. coli isolated from birds infected with avian pathogenic E. coli. A continuous nation wise surveillance of antimicrobial resistance of $E$. coli could be a major step to understand the pattern of resistance and combat treatment failure in poultry. So, with the objective to evaluate antibiotic resistance profile in commercial broilers and layers, this study was designed taking avian pathogenic $E$ coli as a flagship bacterium.

\section{MATERIALS AND METHODS}

This research was conducted in Veterinary Teaching Hospital (VTH) and postgraduate laboratory of Department of Veterinary Medicine, Agriculture and Forestry University (AFU) of Nepal during 2014 to January 2016.

\section{Study population}

All those birds dead or live which were brought to veterinary teaching hospital for clinical and post mortem examination were defined as study population.

\section{Clinical and PM examination, Inclusion criteria and Sample collection}

First, a thorough clinical examination of the birds was conducted with special focus on clinical symptoms of colibacillosis such as swollen head, swollen eyelid, diarrheic vent, swollen joints etc. If the birds were live, they were sacrificed by detaching atlato-axial joint through swirling of neck. A team of registered and experienced veterinarians conducted post mortem examination (PME) of dead birds. Air sacculitis, fibrinous pericarditis, fibrinous perihepatitis, and coligranuloma were major criteria for presumptive diagnosis of colibacillosis on PME. Those layer/broiler birds which were not taking antibiotic orally or parenterally forlast 2 weeks before they were brought to hospital and diagnosed with collibacillosis on PME were included in sampling frame. The first 40 birds for both broiler and layer type chicken totaling 80 birds that fulfilled the inclusion criteria were selected to collect liver samples during PME. Here, each bird in the sampling frame represented a commercial farm. All necessary information on daily management practices and previous treatments were obtained from farmer's record book, sheets or face to face interview.

The reason to take equal number of sample for both broilers and layers was to make a comparison. During postmortem examination, the liver samples were collected aseptically and dispatched to Post graduate laboratory of Department of Veterinary Medicine and Microbiology Unit of Veterinary Teaching Hospital, AFU for culture, isolation and necessary biochemical tests. 


\section{Bacteriological Examination}

The surface of liver sample in petriplate was first sterilized with red hot spatula. The redhot inoculating loop was first cooled on the sterilized surface of liver and then inserted gently into liver to take a loopful of content as inoculum. The inoculum was cultured in MacConkey agar and Eosine Methylene Blue (EMB) (HiMedia, India). The quadrant streak method, using whole plate, was employed for culture. After inoculation, the media was incubated for at least 24 hours at $37^{\circ} \mathrm{C}$ and colony was observed. Pink colony on MacConkey and metallic blue sheen in EMB agar were suggestive of E. coli. Colonies showing typical $E$. coli characteristics and morphology were transferred to nutrient agar and incubated at $37{ }^{\circ} \mathrm{C}$ for 24 hours. A single colony was used for biochemical test and confirmation. Confirmation of $E$. coli was drawn by positive Indole test, positive Methyl Red test, negative Voges Praskaur Test, and negative Citrate utilization test [IMViC test (+ + - -)] (Quinn et al., 1984, and Prescott \& Dowling, 2013). In indole test, pink/red color on the top of broth indicates a positive result for E. coli. Methyl red (MR) and Voges Proskauer (VP) test used MRVP broth. Appearance of red color in MR test and pinkish red color in VP test indicates positive for E. coli. Appearance of blue color in citrate agar means positive test for E. coli.

\section{Antimicrobial susceptibility testing}

The isolates of avian pathogenic $E$. Coli from nutrient agar were subjected for antimicrobial susceptibility test (AST) for study of resistance pattern according to protocol of National Committee for Clinical Laboratory Standards (NCCLS, 2003). The in vitro antimicrobial susceptibility pattern of $E$. coli was determined in Muller Hinton agar plates by Kirby-Bauer disc diffusion method. For this, at least 20 pure colonies of E. coli were taken directly from nutrient agar plate to make a suspension of $1 \mathrm{ml}$ normal saline. The colony was mixed well in the vortex mixture and a slight turbid color was obtained. The suspension was streaked using a sterile, non-toxic swab on an applicator stick, in three directions over the entire surface of Muller-Hinton media (Hi-media, India) to obtain uniform inoculation. The antimicrobial disc (HiMedia, India) was placed onto the agar surface gently using sterile forceps and kept no closer than $24 \mathrm{~mm}$ (center to center) which is equivalent to 6 discs per standard $90 \mathrm{~mm}$ petri-dish. The medium was incubated aerobically at $37^{\circ} \mathrm{C}$ for 24 hours. The degree of resistance or susceptibility was studied based on zone of inhibition, and interpretation was inferred as mentioned in (Table 1).

The selection of antimicrobial molecules was based on their frequency and volume of use as additive in compound feed and while treating colibacilosis in poultry. This information was obtained from registered veterinarians working in poultry industries in Chitwan district, veterinary teaching hospital of AFU, avian laboratories, and veterinary drug centers in Chitwan district through either face to face interview or database analysis of veterinary teaching hospital of AFU. The antimicrobial molecules selected were given in (Table 1). 
Table 1: Antimicrobials and their concentration tested in disc diffusion method, and their interpretation criteria

\begin{tabular}{llllll}
\hline $\begin{array}{l}\text { Antimicrobial } \\
\text { disc }\end{array}$ & $\begin{array}{l}\text { Abbrevi } \\
\text { ation }\end{array}$ & $\begin{array}{l}\text { Concentration } \\
\text { /disc }\end{array}$ & $\begin{array}{l}\text { Interpretation criteria of } \\
\text { inhibition (mm) }\end{array}$ & zone of \\
\cline { 3 - 5 } & & Resistance & Intermediate & Sensitive \\
\hline Amoxycillin & $\mathrm{AC}$ & $20 \mu \mathrm{g}$ & $\leq 13$ & $14-17$ & $\geq 18$ \\
Colistin Sulphate & $\mathrm{CL}$ & $10 \mu \mathrm{g}$ & $\leq 10$ & & $\geq 11$ \\
Cephalexin & $\mathrm{CFX}$ & $5 \mu \mathrm{g}$ & $\leq 15$ & $16-18$ & $\geq 19$ \\
Chloramphenicol & $\mathrm{C}$ & $30 \mu \mathrm{g}$ & $\leq 12$ & $13-17$ & $\geq 18$ \\
Tetracycline & $\mathrm{T}$ & $30 \mu \mathrm{g}$ & $\leq 14$ & $15-18$ & $\geq 19$ \\
Ciprofloxacin & $\mathrm{CF}$ & $5 \mu \mathrm{g}$ & $\leq 15$ & $16-20$ & $\geq 21$ \\
Enrofloxacin & $\mathrm{EN}$ & $10 \mu \mathrm{g}$ & $\leq 14$ & $15-17$ & $\geq 18$ \\
Livofloxacin & $\mathrm{LE}$ & $5 \mu \mathrm{g}$ & $\leq 13$ & $14-16$ & $\geq 17$ \\
Gentamicin & $\mathrm{G}$ & $10 \mu \mathrm{g}$ & $\leq 12$ & $13-14$ & $\geq 15$ \\
Amikacin & $\mathrm{AK}$ & $30 \mu \mathrm{g}$ & $\leq 14$ & $15-16$ & $\geq 17$ \\
\hline
\end{tabular}

\section{Statistical analysis}

The required data were collected based on clinical signs, post mortem examination of live or dead birds, culture, isolation and antimicrobial susceptibility test. All descriptive statistics were presented as frequency and percentage. The resistance profile was described in frequency and proportion. The association of antimicrobial resistance of $E$ coli with other factors was studied through Pearson's chi square test and Fisher's exact test (for the frequency less than 5). The data analysis was done using SPSS 18.0 version.

\section{RESULTS}

The result showed that most of the commercial birds brought to hospitals were Hy-line, Lohman, and H\& N for laying hen types and Vancobb- 500 for broiler types. Swollen head syndrome, lameness, synovitis were the characteristic clinical signs and post mortem lesions for diagnosis of colibacillosis. The recognition of presence of these clinical signs and post mortem lesions in in broilers or layers was based on the knowledge and experience of Veterinarians in the research team and standard photographs of lesions. The distribution of these clinical signs and post mortem lesions are given in (Table 2). Significantly, higher proportion of broiler birds were found to suffer from swollen head syndrome, lameness, synovitis compared to that of layer birds. Omphalitis was significantly higher in broiler types, however, coligranuloma was significantly higher in laying hen type birds (Table 2).

The association of type of bird (broilers or layers) with presence or absence of characteristics clinical signs and post mortem lesion (one by one) was studied through Pearson's chi-square test (a $2 \times 2$ contingency table for each sign and lesion). The degree of freedom was $1[(2-1) \times(2-1)=1]$. 
Table 2: Distribution of clinical signs and postmortem lesions in birds (Broilers and Layers)

\begin{tabular}{llll}
\hline \multirow{2}{*}{ Clinical signs } & Bird type & $\begin{array}{l}\text { Pearson's } \chi^{2}-\text { test } \\
\text { p value }\end{array}$ \\
\cline { 2 - 4 } & Broiler $(\mathbf{n}=\mathbf{4 0})$ & Layers $(\mathbf{n = 4 0 )}$ & 0.00 \\
Swollen head & $21(52.5)$ & $5(12.5)$ & $0.01^{*}$ \\
Lameness & $17(42.5)$ & $3(7.5)$ & $0.03^{*}$ \\
Synovitis & $11(27.5)$ & $1(2.5)$ & $\mathrm{NA}$ \\
Post-mortem lesions & & & 0.26 \\
Omphalitis & $16(40.0)$ & $0(0)$ & 0.63 \\
$\begin{array}{l}\text { Peritonitis } \\
\text { Fibrinous pericarditis }\end{array}$ & $17(42.5)$ & $23(57.5)$ & \\
Fibrinous perihepatitis & $34(85.0)$ & $38(95.0)$ & $0.02^{*}$ \\
Coligranuloma & $31(77.5)$ & $31(77.5)$ & $9(22.5)$ \\
\hline
\end{tabular}

The value in parentheses are in percent (within the bird type). * Fischer's exact test

$\mathrm{NA}=$ The omphalitis was not compared between broilers and layers because it is the characteristics post mortem lesion on broilers only but not in layers. So, there were no any omphalitis in laying hens.

\section{Antimicrobial resistance pattern in poultry}

The antibiogram study showed that avian pathogenic E. coli isolated from layers had developed resistance to several antibiotic molecules in higher proportion compared to that of broilers. In addition, the avian pathogenic $E$. coli showed relatively higher resistance to cephalexin and amoxicillin compared to other antibiotics both in broilers and laying hen birds. However, we found that $E$. coli had not developed resistance to amikacin both in broilers and layers. The interesting finding, we got in this study was that a proportion of $E$. coli isolated from layers had shown resistance to Gentamicin but none of the E. coli isolated from broilers had shown resistance towards Gentamicin (Table 3).

Table 3: Comparison of resistance of $E$ coli to different antibiotic molecules in birds

\begin{tabular}{llll}
\hline Antimicrobial agent & \multicolumn{2}{c}{ Antimicrobial resistance in bird type } & $\begin{array}{l}\text { Pearson's } \chi^{\mathbf{2}} \text { test } \\
\text { p-value }\end{array}$ \\
\cline { 2 - 3 } & Broiler $(\mathbf{n}=\mathbf{4 0})$ & Layer $(\mathbf{n}=\mathbf{4 0})$ & 0.275 \\
\hline Amoxicillin & $30(75.0)$ & $35(87.5)$ & 0.021 \\
Colistin sulphate & $20(50.0)$ & $30(75.0)$ & $\mathbf{0 . 1 3 0}$ \\
Cephalexin & $\mathbf{3 1}(\mathbf{7 7 . 5 )}$ & $\mathbf{3 6}(\mathbf{9 0 . 0 )}$ & 0.012 \\
Chloramphenicol & $19(47.5)$ & $30(75.0)$ & 0.000 \\
Tetracycline & $25(62.5)$ & $38(95.0)$ & 0.007 \\
Ciprofloxacin & $16(40.0)$ & $28(70.0)$ & 0.004 \\
Enrofloxacin & $15(37.4)$ & $28(70.0)$ & 0.059 \\
Levofloxacin & $7(17.5)$ & $16(40.0)$ & $0.006^{*}$ \\
Gentamicin & $0(0)$ & $7(17.5)$ & \\
Amikacin & $0(0)$ & $0(0)$ & \\
\hline
\end{tabular}

The value in parenthesis indicates percent; * Fischer's exact test 
The association of antimicrobial resistance of E. coli with type of bird (broilers or layers) was studied (one by one for each antimicrobial) through Pearson's chi-square test. A $2 \times 2$ contingency table was formed (broilers and layer vs resistance or not resistance). Thus, the degree of freedom was $1[(2-1) \times(2-1)=1]$.

In our study, $E$ coli resistance to Cephalexin was highest $(81 \%, \mathrm{~N}=80)$ followed by Amoxycillin (81\%), Tetracycline (79\%), Colistin sulphate (62\%), Chloramphenicol (61\%), Ciprofloxacin (55\%), Enrofloxacin (54\%), Levofloxacin (29\%), but there was no resistance against amikacin. The $E$. coli isolate which is resistant to two or more than two antimicrobials were considered multidrug resistant isolates. Substantial proportion of $E$. coli were multidrug resistance. The frequency of multidrug resistant $E$. coli isolated from broilers and layers were highest for Amoxicillin-Cephalexin combination, and Tetracycline and Amoxicillin combination respectively. The details of multidrug antimicrobial resistance profile of E. coli isolates are presented in (Table 4).

Table 4: Number of $E$ coli isolates resistance to various antimicrobials

\begin{tabular}{|c|c|c|c|}
\hline $\begin{array}{l}\text { No. of antimicrobials } \\
\text { resistant to which } E \text {. }\end{array}$ & Combination & $\begin{array}{l}\text { No. } \\
\text { isolates }\end{array}$ & $\begin{array}{l}\text { resistan } \\
\text { E. coli }\end{array}$ \\
\hline & & Broiler & Layers \\
\hline 2 & $\begin{array}{l}\text { AC-CFX (Broiler) } \\
\text { AC-T (Layer) }\end{array}$ & 30 & 34 \\
\hline 3 & & 22 & 31 \\
\hline 4 & AC-CFX-T-C & 17 & 26 \\
\hline 5 & AC-CFX-T-C-CL & 14 & 23 \\
\hline 6 & AC-CFX-T-C-CL-CF & 9 & 17 \\
\hline 7 & $\begin{array}{l}\text { AC-CFX-T-C-CL-CF-LE (Broiler) } \\
\text { AC-CFX-T-C-CL-CF-EN (Layer) }\end{array}$ & 2 & 8 \\
\hline 8 & AC-CFX-T-C-CL-CF-LE-G & 0 & 1 \\
\hline 9 & AC-CFX-T-C-CL-CF-EN-LE-G & 0 & 0 \\
\hline 10 & AC-CFX-T-C-CL-CF-EN-LE-G-AK & 0 & 0 \\
\hline
\end{tabular}

The resistance profile of $E$. coli for combination with highest frequency are mentioned only. For eg: E. coli had highest no of resistance for combination of two antimicrobials (Amoxycillin and Cephalexin) in broiler and that of tetracycline and Cephalexin in layers.

\section{DISCUSSION}

The avian pathogenic E. coli showed the highest level of resistance to cephalexin in both broiler and layer type birds. The antimicrobial resistance of $E$. coli isolated from layers showed a significant higher proportion for colistin sulphate $(\mathrm{p}<0.05)$, chloramphenicol $(\mathrm{p}<$ $0.05)$, tertracycline $(p<0.001)$, ciprofloxacin $(p<0.01)$, enrofloxacin $(p<0.05)$ and gentamicin $(\mathrm{p}<0.01)$ in comparison to that of broilers. All the E. coli isolated from broilers were sensitive for gentamicin whereas $17.5 \%(\mathrm{n}=7)$ of isolates in layers had developed resistance to it. E. coli isolates from layers mostly showed resistant for most of the antibiotic molecules. The high resistance to several antibiotics in layers might be due to high level of selective pressure because of continuous use of antibiotics since long time in 
feed. The continuous use of antimicrobial agent creates a selective pressure on bacteria to emerge as a resistant strain (Tenvor, 2006). Dibner \& Richards (2005) discussed an epidemiological linkage on prophylactic use of antimicrobials in animal feed and emergence of resistance. World Health Oganization (2000) suggested in the report that animal health management should be routinely practiced avoiding prophylactic use of antimicrobials to as to take precaution to prevent antibiotic resistance. All these reports and finding underpins that the continuous use of antimicrobials is one of the causes to develop resistance in bacteria. This finding coincides with the findings of Karczmarczyk, et al., (2011) where resistance to Tetracycline was the highest followed by Cephalothin (cephalosporin), the Amoxicillin. This finding is also partly supported by the findings of Saidi et al., (2012) where E. coli isolates showed moderate rates of resistance to tetracycline and chloramphenicol.

This study showed that most of $E$. coli isolates were multiple drug resistant (MDR) which is in the line of Guerra et al., (2003), Miranda et al., (2008) and Jiang et al., (2011). Antibiotic resistance of avian bacterial pathogens is also a common problem in poultry in Bangladesh. Hasan et al.,(2011) noted that more than 55\% ( $=101)$ of $E$ coli isolated were resistant to at least one or more of the tested antibiotics, and $36 \%$ of the isolates showed multiple-drug-resistant phenotypes. The most common resistances observed were against Tetracycline. This supports our finding in the way that the resistance of $E$. coli was highest against tetracycline in laying birds. It also reported moderate resistance to gentamicin which is in same line to our finding.

According to Jiang et al., (2011), E. coli strains isolated from both apparently healthy (from feces) and diseased (from liver) poultry (chicken, ducks and partridges) $(\mathrm{n}=389)$ from China had highest resistance to tetracycline (91\%), amoxicillin $(40 \%)$, and Chloramphenicol (34\%). The high rate of resistance towards tetracycline, amoxicillin and chloramphenicol support our finding. They also found substantial proportion of E coli isolates from poultry which were multidrug resistant. Similarly, Zhang et al., (2014) from Hebei of China reported high rate of resistance toward $(\mathrm{N}=111)$ gentamicin $(95 \%)$, amikacin (46\%), which is contrast to this study. This can be because of continuous over exploitation of aminoglycosides in growing and finishing poultry in China, however the drugs antimicrobials in the group of aminoglycosides are not commonly used in poultry feed. Samanta et al., (2013) from India reported prevalence of $E$ coli in healthy layers and their environment. The report showed that the resistance of the isolates was most frequently observed to chloramphenicol $(87 \%, \mathrm{n}=313)$. In their study, none of the isolates was found to possess quinolone resistance which is contrast to our finding where substantial proportion of E. coli have developed resistant to quinolones (ciprofloxacin, levofloxacin). Several reports of multidrug resistant E. coli isolated from apparently healthy or sick chickens or from faecal and liver samples of poultry have been published emanating from African countries (Maine et al., 1998; Geornaras et al., 2001; Oladele et al., 2008,).

One of the most striking factors for antimicrobial resistance in poultry is the haphazard use of antibiotics. The other factors which promotes this resistance might be poor sanitation of barn and hence the crowding of several bacteria in gastrointestinal tract of 
poultry. The higher level of antibiotic resistance in laying hens could be because of continuous use of antibiotics for longer time compared to that of broilers. All the laying hens in the samples were more than 20 weeks of age and they have been provided with antibiotics in feed since day one old. The higher level of resistance to Amoxicillin and Tetracycline shown in the study could be because of higher use of these antimicrobial molecules in poultry either in feed or via drinking water. Amoxicillin and tetracycline are the most commonly used antibiotic molecules for treatment of poultry in Nepal. Most of the researchers Miranda et al., (2008); Rosengren(2008) and Varga et al., (2009) and Jiang et al., (2011) agreed that there is a direct relationship between antimicrobial resistance and antimicrobial use. The layers birds have longer period of growth relative broilers which results in consumption of higher quantities of a wider range of antimicrobials by laying hens. This could be a reason for higher proportion of antimicrobial resistance of $E$. coli to various antimicrobials (Jianget al., 2011).

The higher proportion of resistant bacteria towards amoxicillin and tetracycline possess a chance of spilling over to other population. These bacteria can transmit the resistance gene to other bacteria to develop the resistance for antimicrobials (Alekshun and Levy, 2007). Till date, the resistance towards amikacin has not been reported in poultry, however, if the use of antibiotic molecules increases in the increasing order, some days in future, the bacteria in poultry can develop resistance substantially even to gentamicin and amikacin. The intestinal flora of poultry can provide a reservoir of antibiotic resistant bacteria that can infect or colonize humans via the food chain.

\section{CONCLUSION}

The E. coli isolated from broilers were highly resistant to cephalexin followed by amoxicillin, tetracycline, and chloramphenicol. The $E$ coli isolated from layers were highly resistant to Tetracycline followed by Cephalexin, Amoxicillin, Ciprofloxacin, Enrofloxacin etc. The E. coli isolated from both broilers and layers were sensitive to amikacin. E. coli isolated from broilers were not resistant to Gentamicin, however, some proportion of $E$. coli isolated from layers were resistant to Gentamicin. The level of antimicrobial resistance was found higher in $E$. coli isolated from layers relative to broilers. A substantial proportion E. coli isolated from both broilers and layers suffered from colibacillosis showed a multi-drug antimicrobial resistance pattern.

\section{ACKNOWLEDGEMENT}

The Directorate of Research (DOR) of AFU is highly acknowledged for funding this research. Our sincere thanks are extended to laboratory personals Anjan Adhikary, Deepak Adhikari, Deependra Pokhrel and Ves Bahadur for their tireless support during sample collection, culture, isolation, and antibiogram during research tenure. 


\section{REFERENCES}

A. (2008). Antimicrobial resistance of fecal Salmonella spp. isolated from all phases of pig production in 20 herds in Alberta and Saskatchewan. Canadian Journal of Veterinary Research, 72(2): 151.

Agabou, A., Lezzar, N., Ouchenane, Z., Khemissi, S., Satta, D., Sotto, A., Lavigne, J.P. and Pantel, A. (2016). Clonal relationship between human and avain ciprofloxacin-resistant Escherichia coli isolates in North-Eastern Algeria. European Journal of Clinical Microbiology and Infectious Diseases, 35(2): 227-234.

Alekshun, M.N. and Levy, S.B. (2007). Molecular mechanisms of antibacterial multidrug resistance. Cell, 128(6): 1037-1050.

Aryal, S. (2001). Antibiotic resistance: A Concern to Veterinary and Human Medicine. Nepal Agriculture Research Journal, 4:66-70.

Baral, P., Neupane, S., Marasini, B.P., Ghimire, K.R., Lekhak, B. and Shrestha, B. (2012). High prevalence of multidrug resistance in bacterial uropathogens from Kathmandu, Nepal. BMC Research Notes, 5(1): 38.

Börjesson, S., Guillard, T., Landén, A., Bengtsson, B. and Nilsson, O. (2016). Introduction of quinolone resistant Escherichia coli to Swedish broiler population by imported breeding animals. Veterinary microbiology, 194: 74-78.

Diarra, M.S., Silversides, F.G., Diarrassouba, F., Pritchard, J., Masson, L., Brousseau, R. and Topp, E. (2007). Impact of feed supplementation with antimicrobial agents on growth performance of broiler chickens, Clostridium perfringens and Enterococcus counts, and antibiotic resistance phenotypes and distribution of antimicrobial resistance determinants in Escherichia coli isolates. Applied and environmental microbiology, 73(20): 65666576.

Dibner, J.J. and Richards, J.D. (2005). Antibiotic growth promoters in agriculture: history and mode of action. Poultry science, 84(4): 634-643.

Ewers, C., Antão, E.M., Diehl, I., Philipp, H.C. and Wieler, L.H. (2009). Intestine and environment of the chicken as reservoirs for extraintestinal pathogenic Escherichia coli strains with zoonotic potential. Applied and Environmental Microbiology, 75(1): 184192.

Geornaras, I., Hastings, J.W. and von Holy, A. (2001). Genotypic analysis of Escherichia coli strains from poultry carcasses and their susceptibilities to antimicrobial agents. Applied and environmental microbiology, 67(4): 1940-1944.

Hasan, B., Faruque, R., Drobni, M., Waldenström, J., Sadique, A., Ahmed, K.U., Islam, Z., Parvez, M.B., Oslam, B. and Alam, M. (2011). High prevalence of antibiotic resistance in pathogenic Escherichia coli from large-and small-scale poultry farms in Bangladesh. Avian diseases, 55(4): 689-692.

Jiang, H.X., Lü, D.H., Chen, Z.L., Wang, X.M., Chen, J.R., Liu, Y.H. and Zeng, Z.L. (2011). High prevalence and widespread distribution of multi-resistant Escherichia coli isolates in pigs and poultry in China. The veterinary journal, 187(1): 99-103.

Karczmarczyk, M., Walsh, C., Slowey, R., Leonard, N. and Fanning, S. (2011). Molecular characterization of multidrug-resistant Escherichia coli isolates from Irish cattle farms. Applied and environmental microbiology, 77(20): 7121-7127. 
Khatiwada, R.K. (2011). Country report: Nepal. Department of Livestock Service, Kathmandu. Available on http://www.aphca.org/Events/36th_ APHCA Session/ Papers/ Country Report Nepal.pdf [Assessed on June 2016, 2015].

Manie, T., Khan, S., Brözel, V.S., Veith, W.J. and Gouws, P.A. (1998). Antimicrobial resistance of bacteria isolated from slaughtered and retail chickens in South Africa. Letters in applied microbiology, 26(4): 253-258.

Miranda, J.M., Guarddon, M., Vázquez, B.I., Fente, C.A., Barros-Velazquez, J., Cepeda, A. and Franco, C.M. (2008). Antimicrobial resistance in Enterobacteriaceae strains isolated from organic chicken, conventional chicken and conventional turkey meat: A comparative survey. Food control, 19(4): 412-416.

National committee for clinical laboratory standards. (2003).Performance standards for antimicrobial disk and dilution susceptibility tests for bacteria isolated from animals; Approved standard. NCCLS, M31-A2, (2nd eds.), Wayne, USA.

Oladele Ogunleye, A., Atanda Oyekunle, M. and Olanrewaju Sonibare, A. (2008). Multidrug resistant Escherichia coli isolates of poultry origin in Abeokuta, South Western Nigeria. Veterinarski Arhiv, 78(6): 501-509.

Prescott, J.F. and Dowling, P.M. (Eds.). (2013). Antimicrobial therapy in veterinary medicine. John Wiley \& Sons.

Quinn, P.J., Carter, M.E., Markey, B.K. and Carter, G.R. (1984). Clinical Veterinary Microbiology, Mosby imprint, Elsevier; p. 684.

Rosengren, L. B., C. L. Waldner, R. J. Reid-Smith, S. L. Checkley, M. McFall, and A. Rajic, (2008): Antimicrobial resistance of fecal Salmonella spp. isolated from all phases of pig production in Alberta and Saskatchewan. Can. J. Vet. Res. 72: 151-159.

Saidi, B., Mafirakureva, P. and Mbanga, J. (2012). Antimicrobial resistance of Escherichia coli isolated from chickens with colibacillosis in and around Harare, Zimbabwe. Avian diseases, 57(1): 152-154.

Samanta, I., Joardar, S.N., Das, P.K., Das, P., Sar, T.K., Dutta, T.K. and Isore, D. P. (2013). Virulence repertoire, characterization, and antibiotic resistance pattern analysis of Escherichia coli isolated from backyard layers and their environment in India. Avian diseases, 58(1): 39-45.

Sharma, A.R., Bhatta, D.R., Shrestha, J. and Banjara, M.R. (2013). Antimicrobial susceptibility pattern of Escherichia coli isolated from uninary tract infected patients attending Bir Hospital. Nepal Journal of Science and Technology, 14(1): 177-184.

Shrestha, A., Regmi, P., Dutta, R.K., Khanal, D.R., Aryal, S.R., Thakur, R.P. and Singh, U.M. (2010). First report of antimicrobial resistance of Salmonella isolated from poultry in Nepal. Veterinary microbiology, 144(3): 522-524.

Singh, A., Khan, M.S.R., Saha, S., Hassan, J. and Roy, U. (2013). Isolation and Detection of Antibiotic Sensitivity Pattern of Escherichia coli from Ducks in Bangladesh and Nepal. Microbes and Health, 1(1): 6-8.

Tenover, F.C. (2006). Mechanisms of antimicrobial resistance in bacteria. The American journal of medicine, 119(6): S3-S10.

Timoney, J.F., Gillespie, J.H., Scott, F.W. and Barlough, J.K. (1988). The genetics of virulence and antibiotic resistance. In: Hagan and Bruner's Microbiology and infectious diseases of domestic animals. (Eds 8). Cornell University Press, Ithaca and London; Pp.24-32. 
Varga, C., Rajić, A., McFall, M.E., Reid-Smith, R.J., Deckert, A.E., Checkley, S.L. and McEwen, S.A. (2009). Associations between reported on-farm antimicrobial use practices and observed antimicrobial resistance in generic fecal Escherichia coli isolated from Alberta finishing swine farms. Preventive veterinary medicine, 88(3): 185-192.

Zhang, F.Y., Huo, S.Y., Xie, R., Li, Y.R., Wu, X.J., Chen, L.G. andGao, Y.H. (2014). A survey of the frequency of aminoglycoside antibiotic resistant genotypes and phenotypes in Escherichia coli in broilers with septicemia. British Poultry Science, 21(5): 45-52. 